\title{
On the existence and stability of traversable wormhole solutions in modified theories of gravity
}

\author{
Oleksii Sokoliuk $^{1,2, a}{ }_{\mathbb{D}}$, Alexander Baransky ${ }^{1, \mathrm{~b}}$ \\ ${ }_{1}^{1}$ Astronomical Observatory, Taras Shevchenko National University of Kyiv, 3 Observatorna St., Kyiv 04053, Ukraine \\ ${ }^{2}$ Astronomical Observatory of the National Academy of Sciences of Ukraine (MAO NASU), Kyiv 03143, Ukraine
}

Received: 24 May 2021 / Accepted: 18 August 2021 / Published online: 31 August 2021

(c) The Author(s) 2021

\begin{abstract}
We study Morris-Thorne static traversable wormhole solutions in different modified theories of gravity. We focus our study on the quadratic gravity $f(\mathscr{R})=\mathscr{R}+a \mathscr{R}^{2}$, power-law $f(\mathscr{R})=f_{0} \mathscr{R}^{n}$, log-corrected $f(\mathscr{R})=\mathscr{R}+$ $\alpha \mathscr{R}^{2}+\beta \mathscr{R}^{2} \ln \beta \mathscr{R}$ theories, and finally on the exponential hybrid metric-Palatini gravity $f(\hat{\mathscr{R}})=\zeta\left(1+e^{-\frac{\hat{\mathscr{R}}}{\phi}}\right)$. Wormhole fluid near the throat is adopted to be anisotropic, and redshift factor to have a constant value. We solve numerically the Einstein field equations and we derive the suitable shape function for each MOG of our consideration by applying the equation of state $p_{t}=\omega \rho$. Furthermore, we investigate the null energy condition, the weak energy condition, and the strong energy condition with the suitable shape function $b(r)$. The stability of Morris-Thorne traversable wormholes in different modified gravity theories is also analyzed in our paper with a modified Tolman-Oppenheimer-Voklov equation. Besides, we have derived general formulas for the extra force that is present in MTOV due to the non-conserved stress-energy tensor.
\end{abstract}

\section{Introduction}

A large number of papers on static traversable wormholes have been written in the last decades $[2,11,16,20,24,26,31$, $52,53]$. In fact, wormholes are bridges between two branes, universes, or just connections of two points at the manifold. Generally wormholes are asymptotically flat. There were many proposals for the wormhole models. One of the first models was proposed by [15], and it was called the EinsteinRosen bridge. Einstein-Rosen bridges are vacuum solutions of Einstein field equations, and this type of wormholes are just an internal part of the maximally extended Schwarzschild

\footnotetext{
${ }^{a}$ e-mail: oleksii.sokoliuk@mao.kiev.ua (corresponding author)

be-mail: abaransky@ukr.net
}

black hole metric. A maximally extended metric means that this metric has no boundaries and the geodesic lines of the particle can be laid infinitely far into the future. So if the spacetime is maximally extended, then there must be present the so-called white hole interior. The exterior of the white hole is often called another universe. The white hole and the second universe are needed in order to extend the trajectory of a particle that fell beyond the event horizon of Schwardschild's black hole infinitely far into the future.

In Figure. 1 one can see the Penrose diagram, where $i^{0}$ is the infinitely far spacelike point, $i^{-}$is the infinitely distant past, $i^{+}$is the infinitely distant future point. Thus timelike curves lie from $i^{-}$to $i^{+}$. Therefore, similarly to the light cone, here $\mathscr{I}^{+}$and $\mathscr{I}^{-}$are lightlike infinitely distant future/past. The upper shaded part of the figure is the interior of our universe black hole, with a singularity at $r=0$, and the bottom shaded triangle is respectively the white hole interior of another universe with a singularity at $r=0$. As well, $\mathscr{H}^{+}$is the black hole horizon and $\mathscr{H}^{-}$is the white hole antihorizon, $\Sigma$ is a spacelike geodesic trajectory through both universes (Cauchy surface). Here both universes are just Minkowski manifolds.

After the theoretical prediction of Einstein-Rosen bridges and Schwarzschild wormholes, many astrophysicists and cosmologists have begun to search for the possibility of the existence of traversable wormholes. One of the first wormhole options, and at the moment one of the most plausible, is the option proposed by [31]. This is the static traversable Morris-Throne wormhole. This type of wormhole can connect two points of spacetime, and its throat is located in the bulk (with $d_{\text {bulk }}>4$ ). This exact solution is a good definition for a traversable wormhole, but as it turned out, this solution implies the presence of an exotic matter at the throat. Therefore, the Null Energy Condition (NEC) is violated in classical GR gravity. By varying parameter values in modified theories of gravity, we could solve this problem, or at least minimize the amount of NEC violating matter. 


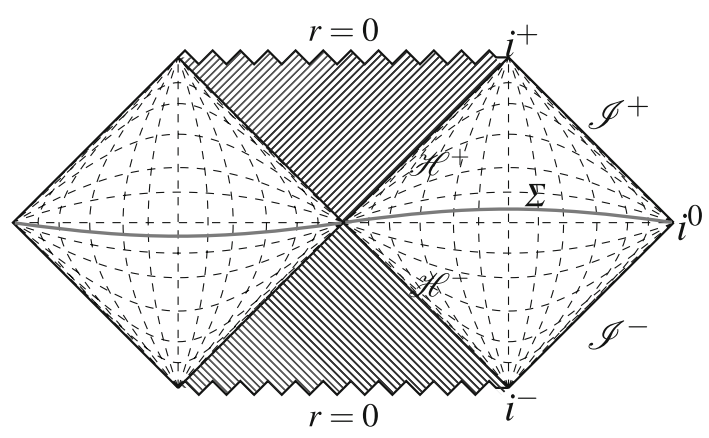

Fig. 1 Penrose diagram for maximally extended Schwarzschild black hole metric (code provided by Robert McNees)

\subsection{Chosen modified theories of gravity to research}

Although the general theory of relativity was and is a wonderful theory that describes our universe well enough, it still has its problems. General theory of relativity (further - GR) is a non-renormalizable theory of gravity, and therefore, can not be conventionally quantized [48]. This is just one of many problems of classical gravity in general relativity. Moreover, GR fails to explain the recent cosmological observations [14]. In order to overpass these problems, astrophysicists introduced in the literature modified theories of gravity. The novelty of the modified theories of gravity is that new geometrodynamic terms are introduced in the gravitational field by the modification of the Einstein-Hilbert (EH) Action Integral. In the following, we briefly introduce the modified theories of gravity of our consideration.

\section{$1.2 f(\mathscr{R})$ gravity}

$f(\mathscr{R})$ gravity is the typical and most popular choice of modified gravity theory, which modifies Einstein-Hilbert action and replaces Ricci scalar in the EH action with arbitrary function of Ricci scalar $f(\mathscr{R})$. The theory was originally proposed in [6]. It has drawn the attention of cosmologists because it can provide a geometric mechanism for the description of inflation $[5,21,49]$ and of the dark energy problem $[7,33]$.

\subsection{Metric-Palatini gravity}

Metric-Palatini is a completely different type of modified gravity theory. In Metric-Palatini gravity in addition to Ricci scalar in EH action function $f(\hat{\mathscr{R}})$ is introduced, which is an arbitrary function for the Palatini scalar, which is constructed from the metric tensor and the Levi-Civita connection. Hybrid Metric-Palatini Gravity (further - HMPG) is a very interesting choice as a modified gravity theory. In particular, HMPG completed some simple and classical tests in the Solar system [9], and also, it was shown that this type of gravity could describe an accelerated universe without dark energy ( $\Lambda$ term) [8]. HMPG and $f(\mathscr{R})$ are fourth-order theories of gravity and they are equivalent to two different scalar-tensor theories. Indeed, there exists a conformal transformation that connects the two theories.

\section{Traversable wormholes in classical GR gravity}

The static non-charged traversable wormhole proper line element, known as Morris-Thorne wormhole is given by the following expression [31]:

$d s^{2}=-e^{2 \Omega(r)} d t^{2}+\frac{1}{1-\frac{b(r)}{r}} d r^{2}+r^{2} d \theta^{2}+r^{2} \sin ^{2} \theta d \phi^{2}$.

Same as Eq. (1), but in Cartesian coordinates the line element becomes

$d s^{2}=-e^{2 \Omega(x)} d t^{2}+\frac{1}{1-\frac{b(x)}{x}} d x^{2}+d y^{2}+d z^{2}$.

Function $\Omega(x)$ is the redshift function and $b(x)$ is so-called wormhole shape function. For GR we have following Action Integral and gravitational Lagrangian $\mathscr{L}_{g}$

$\mathscr{S}_{\mathscr{R}}=\int_{\mathscr{M}} d^{4} x \sqrt{-g} \frac{1}{2} \mathscr{L}_{g}=\int_{\mathscr{M}} d^{4} x \sqrt{-g} \frac{1}{2} \mathscr{R}$,

where we have assumed for the gravitational constant $\kappa=1$. In the latter Action Integral, $g$ is metric tensor determinant: $g=\operatorname{det} g_{\mu \nu}$, and $\mathscr{R}$ is the Ricci scalar of $g$. The Einstein field equations are (Einstein Field Equation or EFE):

$G_{\mu \nu}+\Lambda g_{\mu \nu}=T_{\mu \nu}$,

where $G_{\mu \nu}$ is the Einstein tensor, $\Lambda$ is so-called lambda-term or dark energy (further - DE, in our case we consider universe without $\mathrm{DE}$, so $\Lambda=0), T_{\mu \nu}$ is stress-energy tensor for the additional matter source. The Einstein tensor is defined as follows

$G_{\mu \nu}=R_{\mu \nu}-\frac{1}{2} \mathscr{R} g_{\mu \nu}$.

Here $R_{\mu \nu}$ is the Ricci tensor, $\mathscr{R}$ is the Ricci scalar. So, now we can derive the non-zero components of the Einstein tensor for the line element (1): 


$$
\begin{aligned}
G_{t t}= & -\frac{b^{\prime}(r)}{r^{2}}, \\
G_{r r}= & -\frac{\left(1-\frac{b(r)}{r}\right)\left(2 r b(r) \Omega^{\prime}(r)+b(r)-2 r^{2} \Omega^{\prime}(r)\right)}{r^{2}(r-b(r))}, \\
G_{\theta \theta}= & \frac{\left(r \Omega^{\prime}(r)+1\right)\left(-r b^{\prime}(r)+2 r(r-b(r)) \Omega^{\prime}(r)+b(r)\right)}{2 r^{3}} \\
& +\frac{(r-b(r)) \Omega^{\prime \prime}(r)}{r}, \\
G_{\phi \phi}= & \frac{\left(r \Omega^{\prime}(r)+1\right)\left(-r b^{\prime}(r)+2 r(r-b(r)) \Omega^{\prime}(r)+b(r)\right)}{2 r^{3}} \\
& +\frac{(r-b(r)) \Omega^{\prime \prime}(r)}{r} .
\end{aligned}
$$

In Eqs. (6), (7), (8) and (9) prime $\Omega^{\prime}(r), b^{\prime}(r)$ means total derivative with respect to the independent variable $r$.

\section{EFE's for Morris-Thorne wormholes in different modified theories of gravity}

\section{$3.1 f(\mathscr{R})$ modified gravity case}

In $f(\mathscr{R})$ theory of gravity the EH Action Integral is modified as follows [6]:

$$
\begin{aligned}
\mathscr{S}_{\mathscr{R}}= & \frac{1}{2} \int_{\mathscr{M}}\left(\mathscr{R}+\mathscr{L}_{M}\right) \sqrt{-g} d^{4} x \\
& \Downarrow \\
\mathscr{S}_{f(\mathscr{R})}= & \left.\frac{1}{2} \int_{\mathscr{M}}\left[f(\mathscr{R})+\mathscr{L}_{M}\right)\right] \sqrt{-g} d^{4} x,
\end{aligned}
$$

where $\mathscr{L}_{\mathrm{M}}$ is the matter Lagrangian. In the [47] we already modified general view of the EFE for symmetric metric tensor [48]:

$$
\begin{aligned}
G_{\mu \nu}^{(0)} \equiv R_{\mu \nu}-\frac{1}{2} g_{\mu \nu} \mathscr{R}= & \frac{T_{\mu \nu}^{\mathrm{M}}}{f^{\prime}(\mathscr{R})}+g_{\mu \nu} \frac{\left[f(\mathscr{R})-\mathscr{R} f^{\prime}(\mathscr{R})\right]}{2 f^{\prime}(\mathscr{R})} \\
& +\frac{\left[\nabla_{\mu} \nabla_{\nu} f^{\prime}(\mathscr{R})-g_{\mu \nu} \square f^{\prime}(\mathscr{R})\right]}{f^{\prime}(\mathscr{R})} .
\end{aligned}
$$

Clearly $f^{\prime}(\mathscr{R})$ is the derivative with respect to the Ricci scalar. Furthermore, the stress-energy tensor for the anisotropic fluid is [45]:

$$
T_{\mu \nu}^{\mathrm{M}}=\left(\begin{array}{cccc}
-\rho & 0 & 0 & 0 \\
0 & p_{r} & 0 & 0 \\
0 & 0 & p_{t} & 0 \\
0 & 0 & 0 & p_{t}
\end{array}\right),
$$

where $p_{r}, p_{t}$ are the radial and tangential pressures respectively, and $\rho$ is the energy-density. The parameter for the equation of state (further - EoS) defines the type of matter $\left(\omega=\frac{p}{\rho}\right)$.

\subsubsection{Violation of null energy condition (NEC)}

In GR wormholes are supported by exotic matter, which involves a stress-energy tensor that violates the null energy condition (NEC) $[27,31,51]$. NEC violation in $f(\mathscr{R})$ is given by the following expression:

$$
\begin{aligned}
\rho^{\text {eff }}+p_{r}^{\text {eff }} & =\frac{\rho+p_{r}}{f^{\prime}(\mathscr{R})}+\frac{1}{f^{\prime}(\mathscr{R})} \\
& {\left[\left(f^{\prime}(\mathscr{R})\right)^{\prime \prime}-\left(f^{\prime}(\mathscr{R})\right)^{\prime} \frac{b^{\prime} r-b}{2 r^{2}(1-b / r)}\right] } \\
\Downarrow & \\
\rho^{\text {eff }}+p_{r}^{\text {eff }}= & \frac{b^{\prime} r-b}{r^{3}}<0 .
\end{aligned}
$$

Also, if a wormhole exists, it should obey the following inequalities [27]:

$\frac{f^{\prime}(\mathscr{R}) b^{\prime}}{r^{2}} \geq 0 \Rightarrow \frac{b^{\prime}}{r^{2}}<0 \Rightarrow f^{\prime}(\mathscr{R}) \leq 0$.

Theorem of wormhole non-existence in $f(\mathscr{R})$ gravity is essentially the same the one derived before in [4]:

$\frac{d f(\mathscr{R})}{d \mathscr{R}}<0$

We continue by assuming specific functional form for the $f(\mathscr{R})$ function.

\subsubsection{Derivation of the shape function}

One of the necessary conditions for a wormhole to exist is that wormhole shape function must satisfy EFE's in modified gravity. From Eq. (11) one could derive following EFE's (in general $f(\mathscr{R})$ gravity with arbitrary choice of function) [27]:

$$
\begin{aligned}
\rho & =\frac{f_{R} b^{\prime}}{r^{2}} \\
p_{r} & =-\frac{b f_{R}}{r^{3}}+\frac{f_{R}^{\prime}}{2 r^{2}}\left(b^{\prime} r-b\right)-f_{R}^{\prime \prime}\left(1-\frac{b}{r}\right), \\
p_{t} & =-\frac{f_{R}^{\prime}}{r}\left(1-\frac{b}{r}\right)+\frac{f_{R}}{2 r^{3}}\left(b-b^{\prime} r\right) .
\end{aligned}
$$

Here we already considered wormhole solution without tidal forces, and thus with constant redshift factor. We have chosen this case, because the tidal gravitational forces experienced by a traveler must be bearably small (negligible) [31]. And, therefore with applying equation of state $p_{t}=\omega \rho$ we have 
following equation [27]:

$f_{R}^{\prime}\left(1-\frac{b}{r}\right)-\frac{f_{R}}{2 r^{2}}\left[b-b^{\prime} r(1+2 \omega)\right]=0$.

Hence, from equation above we could find suitable shape function which satisfies EFE's for any kind of $f(\mathscr{R})$ gravity.

\subsubsection{Quadratic gravity $f(\mathscr{R})=\mathscr{R}+\alpha \mathscr{R}^{2}$}

Firstly, we could rewrite Eq. (19) in terms of quadratic MOG:

$$
\begin{aligned}
& \left(1-\frac{b(r)}{r}\right)\left(\frac{4 \alpha b^{\prime \prime}(r)}{r^{2}}-\frac{8 \alpha b^{\prime}(r)}{r^{3}}\right) \\
& -\frac{\left(\frac{4 \alpha b^{\prime}(r)}{r^{2}}+1\right)\left(b(r)-r(2 \omega+1) b^{\prime}(r)\right)}{2 r^{2}}=0 .
\end{aligned}
$$

We solved this equation numerically (there is no possibility to solve this equation algebraically) with initial conditions $b\left(10^{-2}\right)=10^{-3}, b^{\prime}\left(10^{-2}\right)=2 \times 10^{-4}[22]$.

As well, for our MOG shape function, we have following flaring-out condition [23]:

$\frac{\left(b-b^{\prime} r\right)}{b^{2}}>0 \Rightarrow b^{\prime}\left(r_{0}\right)<1$.

We numerically solved Eqs. (20) and (21) on the Figure. 2 . The flaring-out condition was solved for only positive values of $\alpha$, because, as it turned out, with $\alpha<0$ we have that flaring-out condition is violated generally. Thus, in quadratic MOG for positive values of MOG parameter, we have physically acceptable shape function, that satisfies EFE's. Finally, for almost all positive alpha values, the flaring-out condition was satisfied with EoS parameter $\omega=1$ (stiff fluid, presented by [54]) Therefore, we could proceed to the NEC, WEC, and SEC conditions derivation for this MOG, shape function.

\subsubsection{Quadratic gravity energy conditions}

Because of the previously stated reasons (validation of flareout condition for every $\alpha \geq 0$ at the throat), we chose the case with the stiff fluid (as numerical analysis showed, there is no significant differences of energy conditions with different values of EoS parameter in the limit $0<\omega \leq 1)$. Firstly, we could present energy conditions, that we consider in this paper $[1,44]$ :

- Null Energy Condition (NEC): $\rho+p_{r} \geq 0 \wedge \rho+p_{t} \geq 0$

- Weak Energy Condition (WEC): $\rho-p_{r} \geq 0 \wedge \rho+p_{t} \geq 0$

- Strong Energy Condition (SEC): $\rho+p_{r}+2 p_{t} \geq 0$

NEC is a minimal requirement of WEC and SEC conditions and must be obeyed always (if NEC is violated, so-called
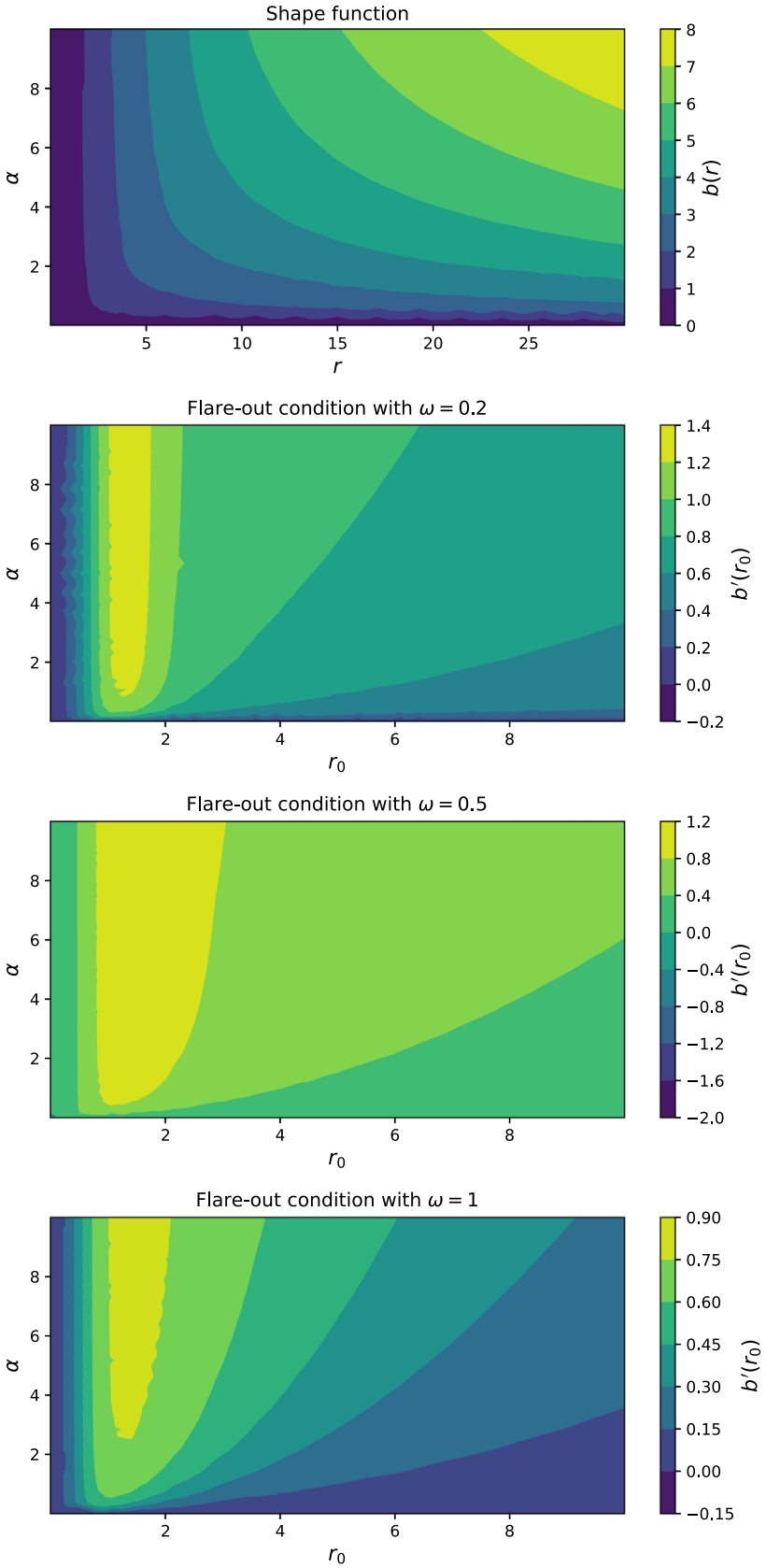

Fig. 2 Numerical solution for (20) and Flaring-out condition validation/violation for positive $\alpha$ parameter in quadratic gravity

exotic matter or in some cases phantom fluid will appear [41]).

We showed numerical solutions for NEC, WEC and SEC energy conditions at Figure. 3. As one may notice, generally NEC is validated for each pressure if $\alpha<1$, as well WEC is validated for both pressure types for any $\alpha>0$. Finally, $\mathrm{SEC}$ is violated. 

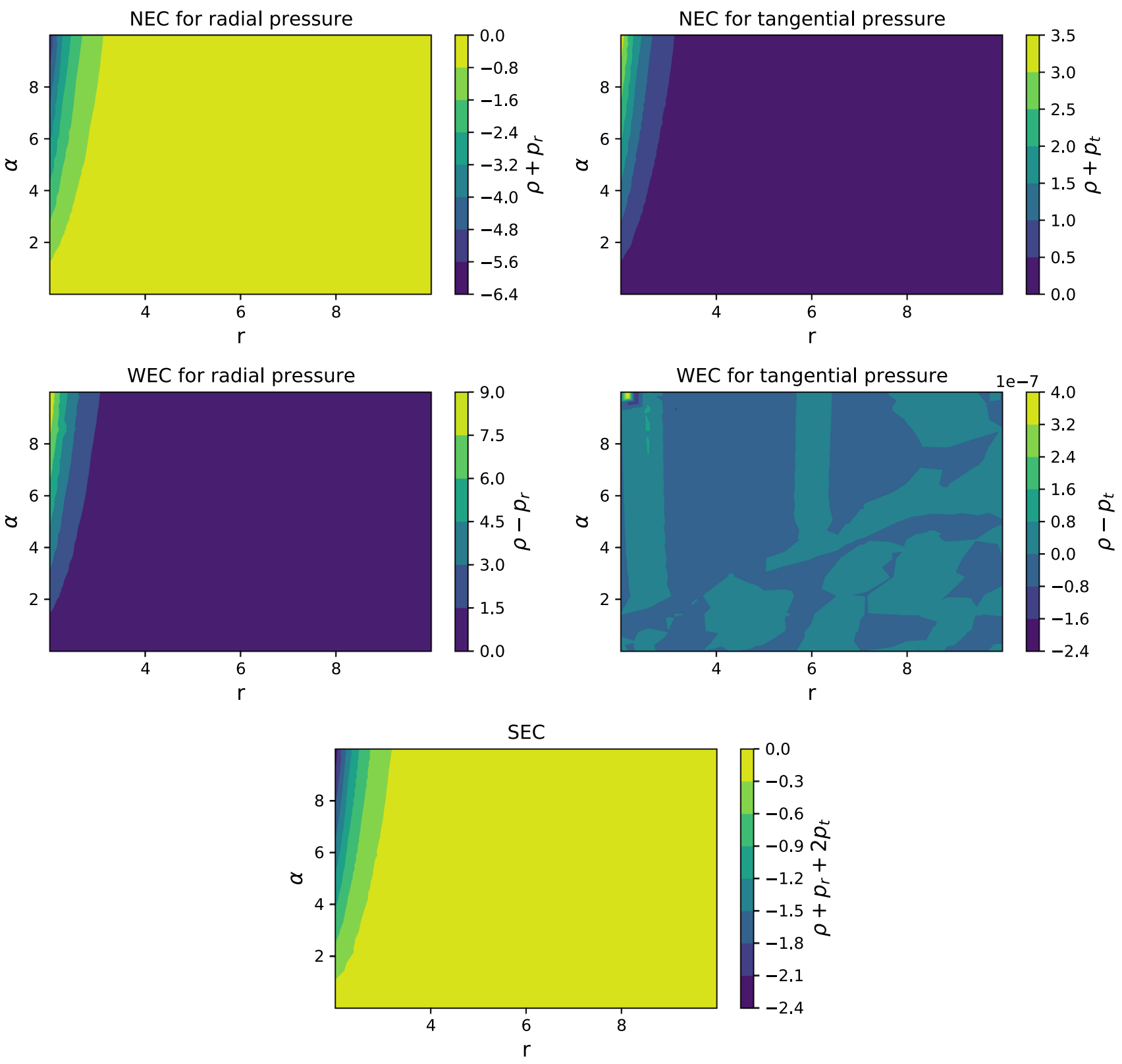

Fig. 3 NEC, WEC and SEC conditions for quadratic gravity with $\omega=1$

\subsubsection{Power law gravity $f(\mathscr{R})=f_{0} \mathscr{R}^{n}$ for $n>1$}

This is another $f(\mathscr{R})$ example of MOG theory, which was described by $[10,34]$. For this type of gravity we have following EH action:

$\mathscr{S}_{f(\mathscr{R})}=\frac{1}{2} \int_{\mathscr{M}}\left[f_{0} \mathscr{R}^{n}\right] \sqrt{-g} d^{4} x$,

where $f_{0}$ is a constant to give correct dimensions to the action and $n$ is the slope parameter [28]. For MOG of this kind we have following form of Eq. (19):

$$
\begin{array}{r}
f_{0} 2^{n-1}(n-1) n\left(1-\frac{b(r)}{r}\right)\left(\frac{b^{\prime}(r)}{r^{2}}\right)^{n-2}\left(\frac{b^{\prime \prime}(r)}{r^{2}}-\frac{2 b^{\prime}(r)}{r^{3}}\right) \\
-\frac{f_{0} 2^{n-2} n\left(\frac{b^{\prime}(r)}{r^{2}}\right)^{n-1}\left(b(r)-r(2 \omega+1) b^{\prime}(r)\right)}{r^{2}}=0 .
\end{array}
$$

On the Fig. 4 we once again plotted numerical solutions for Eqs. (23) and (19) with varying $n /$ constant $\omega$ and varying $\omega /$ constant $n$. As it turned out, the shape function does not depends on the $f_{0}$ parameter. Now that we have decided on the type of shape function for our power-law MOG, we can begin to study the energy conditions of the Morris-Thorne traversable wormhole in the MOG of our consideration. 

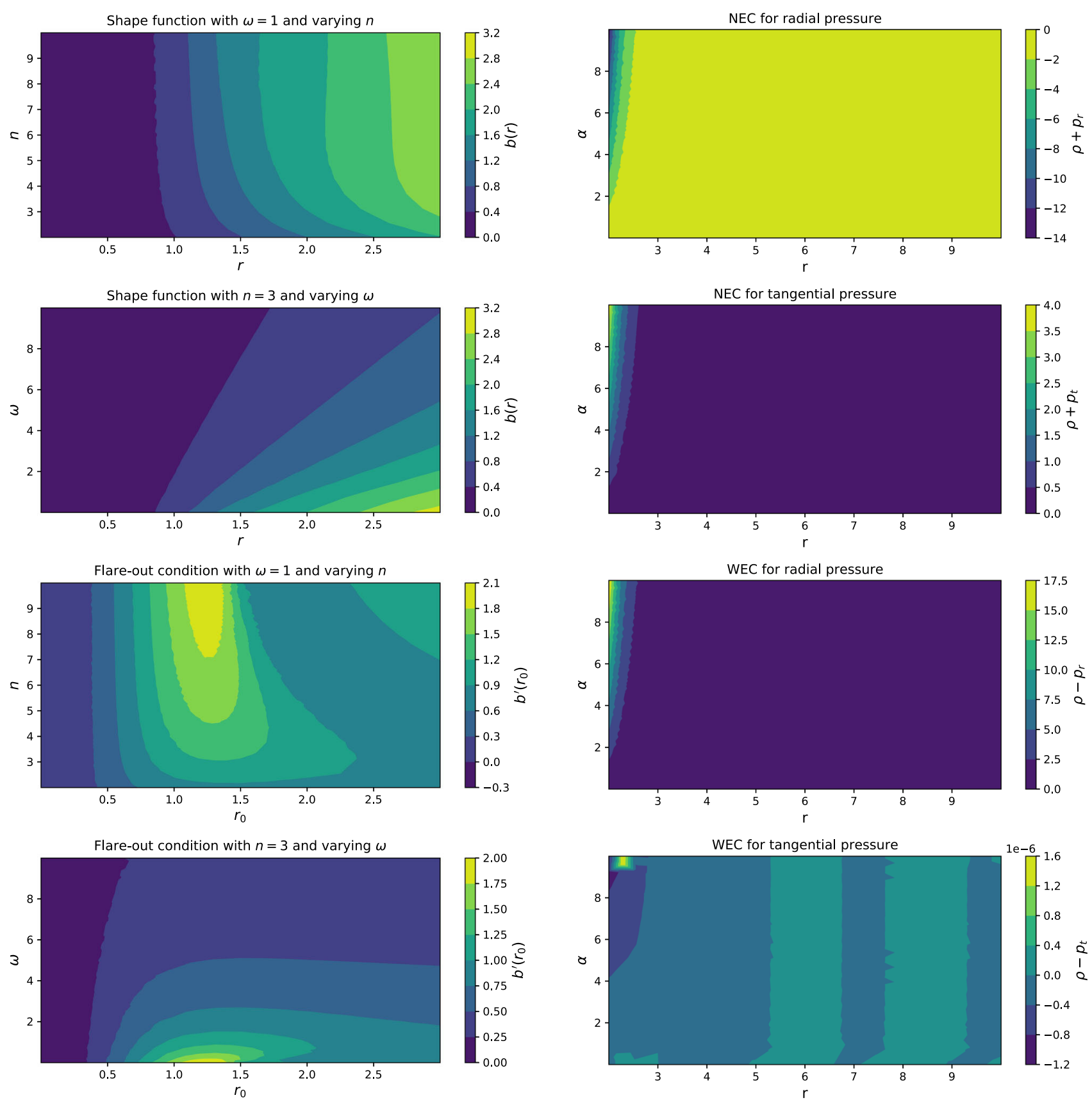

Fig. 4 Numerical solution for Eq. (23) and Flaring-out condition validation/violation for varying $n$ and $\omega$ in power-law gravity

In turn, in the Fig. 5 we illustrated null, weak, and strong energy conditions for power-law gravity. We plotted only one case with $\omega=1$ and $n=3$, because we found that if we will vary these parameters, nothing changes much. Also, it is interesting that our power-law numerical solutions for energy conditions are very similar to those, that we obtained for quadratic MOG (see Fig. 3). In relation, NEC generally

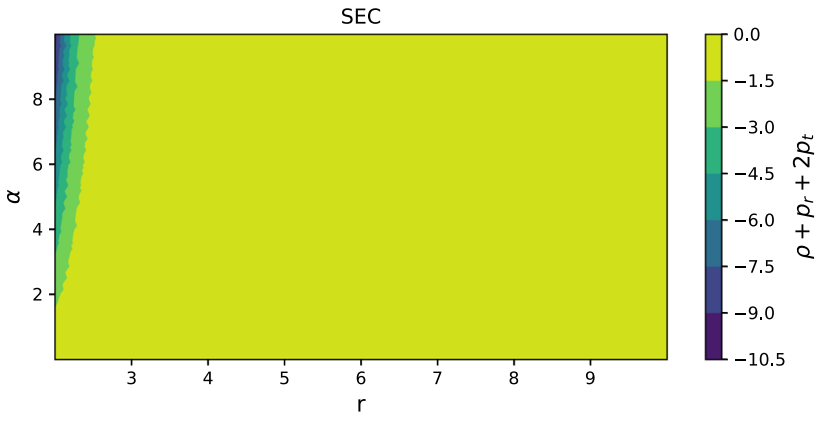

Fig. 5 NEC, WEC and SEC conditions for power-law gravity with $\omega=1$ and $n=3$ 
$\alpha<4$ WEC is validated for both pressure types and finally SEC is violated.

\subsubsection{Logarithmic corrected $f(\mathscr{R})$ gravity}

Logarithmic corrected $f(\mathscr{R})$ gravity was introduced in [40]:

$$
f(\mathscr{R})=\mathscr{R}+\alpha \mathscr{R}^{2}+\beta \mathscr{R}^{2} \log \beta \mathscr{R},
$$

where $\beta>0$ and $\alpha>0$. This type of gravity can describe expanding universe without dark energy [34]. The modified EH Action Integral is defined in this way:

$$
S_{f(\mathscr{R})}=\int_{\mathscr{M}} d^{4} x \sqrt{-g} \frac{1}{2}\left(\mathscr{R}+\alpha \mathscr{R}^{2}+\beta \mathscr{R}^{2} \log \beta \mathscr{R}\right),
$$

Now, with given MOG form we as usual could rewrite Eq. (19) as follows:

$$
\begin{aligned}
& 2(2 \omega+1) b^{\prime}(r)^{2}\left(2 \alpha+2 \beta \log \left(\frac{2 \beta b^{\prime}(r)}{r^{2}}\right)+\beta\right) \\
& +b^{\prime}(r)\left(-8(2 \alpha+3 \beta)-16 \beta \log \left(\frac{2 \beta b^{\prime}(r)}{r^{2}}\right)+r^{2}(2 \omega+1)\right) \\
& +4 r b^{\prime \prime}(r)\left(2 \alpha+2 \beta \log \left(\frac{2 \beta b^{\prime}(r)}{r^{2}}\right)+3 \beta\right) \\
& +b(r)\left(\frac{2 b^{\prime}(r)\left(6 \alpha+6 \beta \log \left(\frac{2 \beta b^{\prime}(r)}{r^{2}}\right)+11 \beta\right)}{r}\right. \\
& \left.-4 b^{\prime \prime}(r)\left(2 \alpha+2 \beta \log \left(\frac{2 \beta b^{\prime}(r)}{r^{2}}\right)+3 \beta\right)-r\right)=0
\end{aligned}
$$

Consequently, on the Fig. 6 we have numerical representation of Eq. (26) and of flare-out condition at the throat. As numerical analysis showed, $b^{\prime}\left(r_{0}\right)<1$ is obeyed for $0<\omega \leq 1 \wedge \alpha>4$ if we assume constant $\beta=1$.

Routinely, for this shape function we placed NEC, WEC and SEC numerical solutions at the Fig. 7. As we found, NEC is violated for radial pressure and validated for tangential, WEC is vice versa validated for radial pressure case and violated for tangential one. Just as with the previous MOG theories, SEC is generally violated.

\subsection{Hybrid metric-Palatini gravity}

The second family of modified theories of our consideration is the HMPG theory, for which the Action Integral is given by $[8,18,37]$ :

$$
\mathscr{S}_{f(\hat{\mathscr{R}})}=\frac{1}{2} \int_{\mathscr{M}} d^{4} x \sqrt{-g}[\mathscr{R}+f(\hat{\mathscr{R}})]
$$
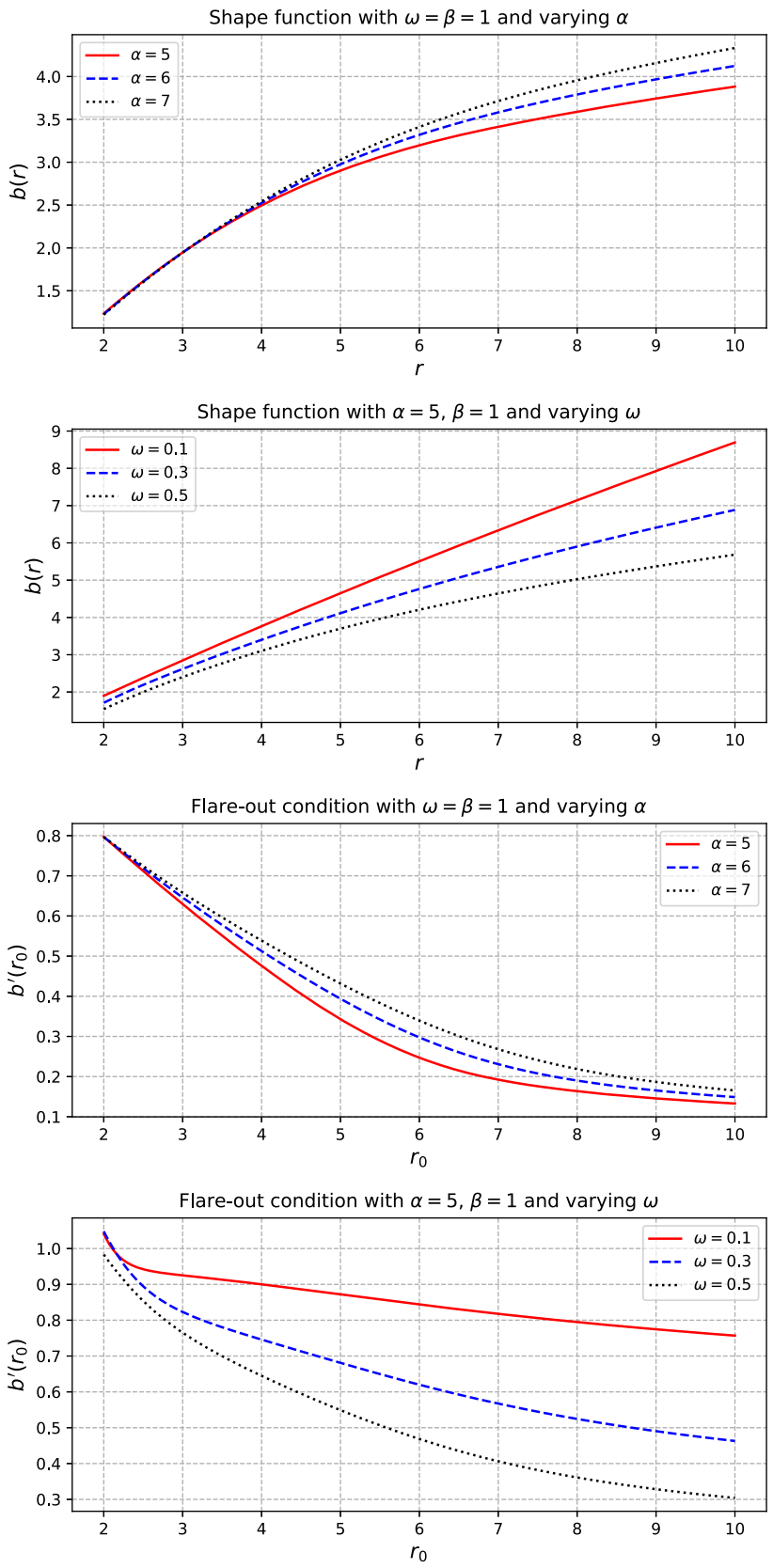

Fig. 6 Numerical solution for Eq. (26) and Flaring-out condition validation/violation for varying $\alpha$ and $\omega$ (we assumed constant $\beta$ for simplicity) in log-corrected gravity

where $\hat{\mathscr{R}}$ is the Palatini scalar, constructed from Palatini curvature tensor, which reads [25]:

$\hat{R}_{\mu \nu}=\hat{R}_{\mu \alpha \nu}^{\alpha}=\hat{\Gamma}_{\mu \nu, \alpha}^{\alpha}-\hat{\Gamma}_{\mu \alpha, \mu}^{\alpha}+\hat{\Gamma}_{\alpha \lambda}^{\alpha} \hat{\Gamma}_{\mu \nu}^{\lambda}-\hat{\Gamma}_{\mu \lambda}^{\alpha} \hat{\Gamma}_{\alpha \nu}^{\lambda}$.

$\hat{\Gamma}_{\mu \nu}^{\alpha}$ is the Levi-Civita connection for metric which is conformal to our wormhole background metric $\left(h_{\mu \nu}=\phi g_{\mu \nu}\right)[3]$. 

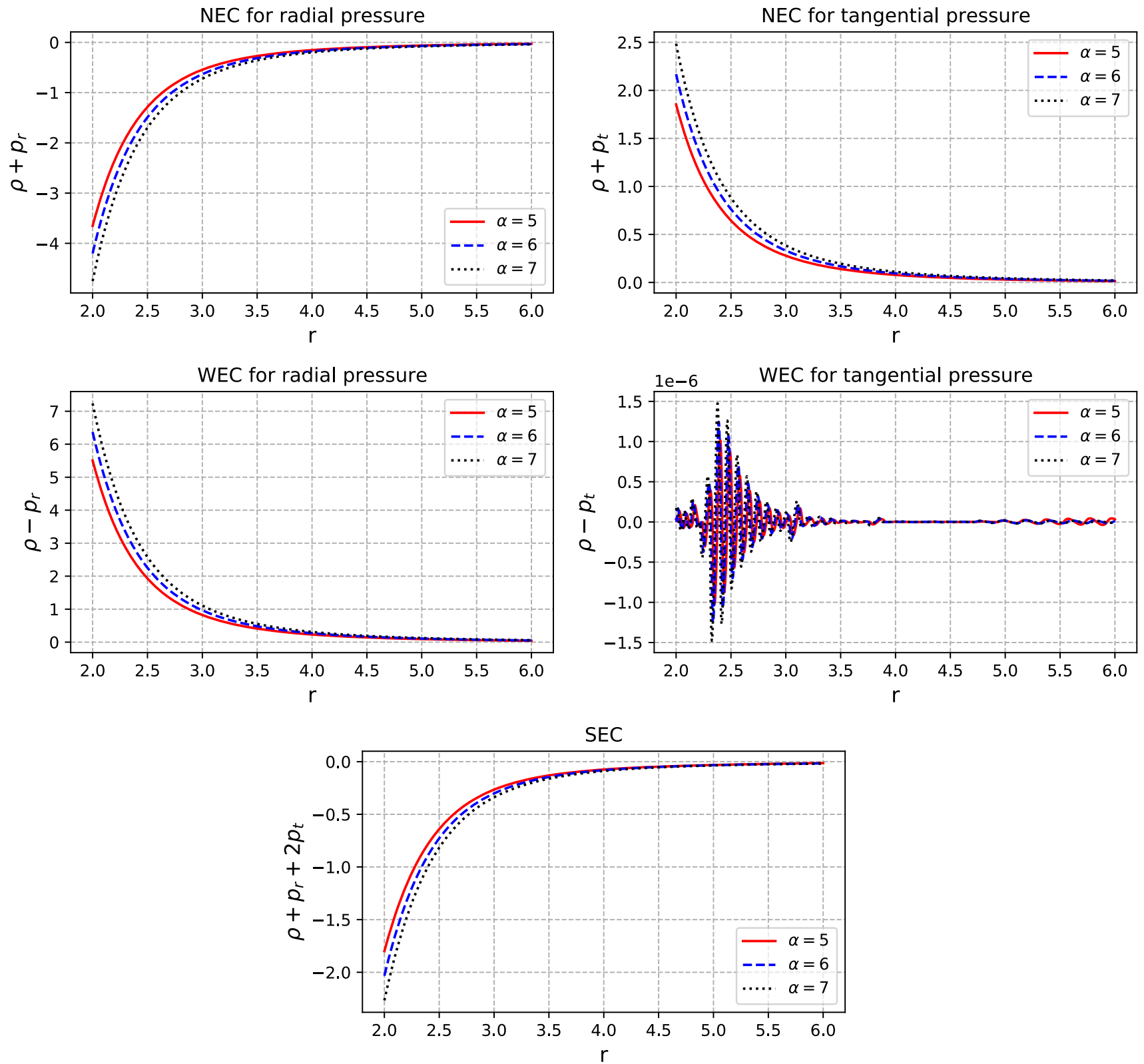

Fig. 7 NEC, WEC and SEC conditions for log-corrected gravity with $\omega=\beta=1$

We could also rewrite Eq. (28) in form [12]:

$\hat{R}_{\mu \nu}=R_{\mu \nu}+\frac{3}{2 \phi^{2}} \partial_{\mu} \phi \partial_{\nu} \phi-\frac{1}{\phi} \nabla_{\mu} \nabla_{\nu} \phi-\frac{1}{2 \phi} g_{\mu \nu} \square \phi$.

Thus for the Palatini scalar we find that

$\hat{\mathscr{R}}=\mathscr{R}+\frac{3}{2 \phi^{2}} \partial_{\mu} \phi \partial^{\mu} \phi-\frac{3}{\phi} \square \phi$.
But, we have as well scalar-tensor representation of hybrid metric-Palatini gravity [19]:

$\mathscr{S}_{f(\hat{\mathscr{R}})}=\frac{1}{2} \int_{\mathscr{M}} d^{4} x \sqrt{-g}\left[(1+\phi) \mathscr{R}+\frac{3}{2 \phi} \partial_{\mu} \phi \partial^{\mu} \phi-V(\phi)\right]$,

where $\phi$ is scalar field and $V(\phi)$ is scalar potential (in metricPalatini gravity scalar field in dynamic [35]). By varying the action, we could obtain following EFE form [19,25]:

$G_{\mu \nu}=\frac{1}{1+\phi} T_{\mu \nu}+T_{\mu \nu}^{(\phi)}$ 


$$
\begin{aligned}
T_{\mu \nu}^{(\phi)}= & \frac{1}{1+\phi}\left[\nabla_{\mu} \nabla_{\nu} \phi-\frac{3}{2 \phi} \nabla_{\mu} \phi \nabla_{\nu} \phi\right. \\
& \left.+\left(\frac{3}{4 \phi} \nabla_{\lambda} \phi \nabla_{\lambda} \phi-\square \phi-\frac{1}{2} V(\phi)\right)\right],
\end{aligned}
$$

where $V(\phi)$ is [3]:

$V(\phi)=\hat{\mathscr{R}} \phi-f(\hat{\mathscr{R}})$,

and scalar field is [12]:

$\phi=\tan ^{2}\left(\sqrt{\frac{3}{8} \bar{\phi}}\right)$.

If $\phi \rightarrow \infty$, then:

$\bar{\phi}=\sqrt{\frac{8}{3}}\left[(-1)^{k} \frac{\pi}{2}+2 k \pi\right], \quad k=0,1,2,3 \ldots$

On the other hand, when $\phi \rightarrow 0$,

$\bar{\phi}=\sqrt{\frac{8}{3}} k \pi$.

If (from Eqs. (47) and (36)) $\phi$ is independent of $x^{\mu}$, then $\hat{\mathscr{R}}=\mathscr{R}$. As we did for $f(\mathscr{R})$ gravity, we could derive energy density and pressures from EFE's:

$$
\begin{aligned}
\rho= & \frac{-2 b^{\prime}(r)-f(\hat{\mathscr{R}}) r^{2}}{2 r^{2}}, \\
p_{r}= & {\left[2(b(r)-r) \cot ^{2}(2 \pi k) b^{\prime}(r)-b(r)\left(f(\hat{\mathscr{R}}) r^{2}\right.\right.} \\
& \left.\left.+2 \cot ^{2}(2 \pi k)+2\right)+f(\hat{\mathscr{R}}) r^{3}\right] /\left[2 r^{2}(r-b(r))\right], \\
p_{t}= & \frac{1}{2}\left(\left[\operatorname { c s c } ^ { 2 } ( 2 \pi k ) \left(r b(r)-b^{\prime}(r)(\cos (4 \pi k)\right.\right.\right. \\
& \left.\left.\left.\left.+r^{2}+1\right)\right)\right] /\left[r^{2}\right]+f(\hat{\mathscr{R}})\right) .
\end{aligned}
$$

Then, $\operatorname{EoS} p_{t} / \rho=\omega$ takes form:

$$
\begin{aligned}
& {\left[\operatorname { c s c } ^ { 2 } ( 2 \pi k ) \left(2 b^{\prime}(r)\left(\cos (4 \pi k)+r^{2}+1\right)-2 r b(r)\right.\right.} \\
& \left.\left.+f(\hat{\mathscr{R}}) r^{2}(\cos (4 \pi k)-1)\right)\right] /\left[4 b^{\prime}(r)+2 f(\hat{\mathscr{R}}) r^{2}\right]=\omega .
\end{aligned}
$$

By solving this equation, we could obtain shape function $b(r)$ in the physically acceptable form (i.e. shape function that satisfies EFE's)
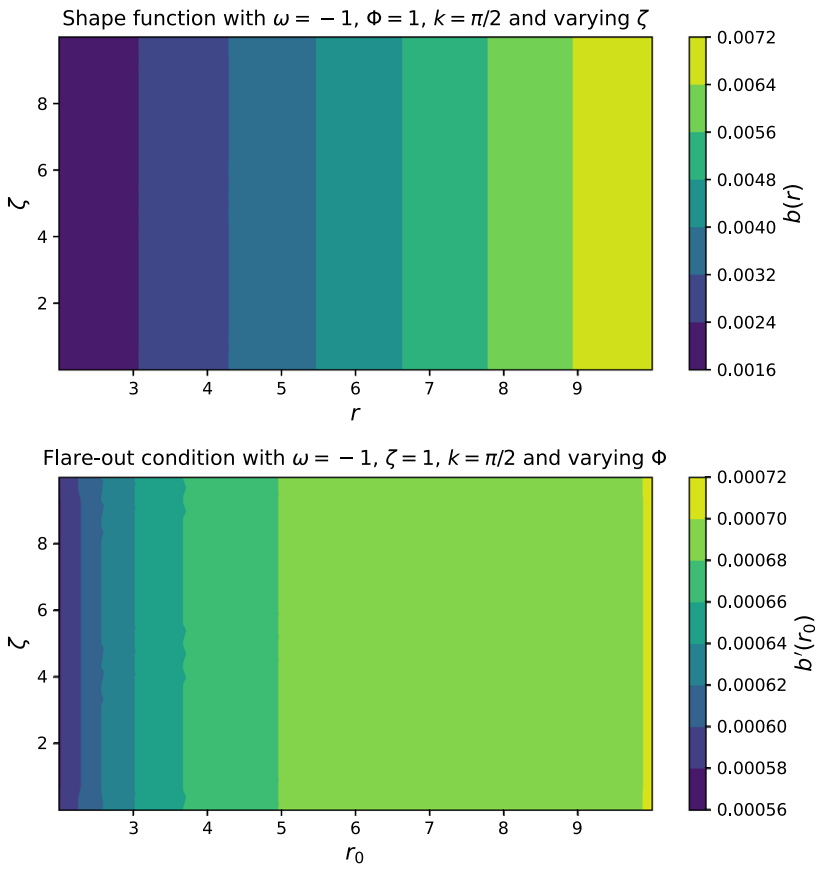

Fig. 8 Shape function and flare-out condition at the throat for HMPG gravity with $\omega=-1$ and $k=\pi / 2$

3.2.1 Exponential $f(\hat{\mathscr{R}})=\zeta\left(1+e^{-\frac{\hat{\mathscr{R}}}{\Phi}}\right)$

In this study we consider the following Palatini-scalar function [17]:

$f(\hat{\mathscr{R}})=\zeta\left(1+e^{-\frac{\hat{\mathscr{R}}}{\Phi}}\right)$

Therefore, we could rewrite EoS (41), that describe shape function, which satisfies EFE's as follows:

$$
\begin{aligned}
& {\left[\csc ^{2}(2 \pi k)\left(r b(r)-b^{\prime}(r)\left(\cos (4 \pi k)+r^{2}+1\right)\right)\right.} \\
& \left.\quad+\zeta r^{2}\left(e^{-\frac{2 b^{\prime}(r)}{r^{2} \Phi}}+1\right)\right] /\left[\zeta r^{2}\left(e^{-\frac{2 b^{\prime}(r)}{r^{2} \Phi}}+1\right)+2 b^{\prime}(r)\right]=\omega
\end{aligned}
$$

Hence, on the Fig. 8 we located the numerical solution for the equation above and proof that in this MOG for our shape function flare-our condition is satisfied. As we saw from the numerical analysis, shape function does not depends on the $\zeta$ MOG free parameter. Also, it is necessary to note that we have only one physically acceptable solution with EoS parameter $\omega=-1$ (dark energy like fluid).

Finally, in the Fig. 9 we illustrated energy conditions for HMPG gravity. As one may notice, NEC is violated for both pressure types, and thus there is always present exotic matter at the throat. WEC condition could be satisfied by assuming that $\zeta<1$, SEC is validated for every $\zeta>0$. 

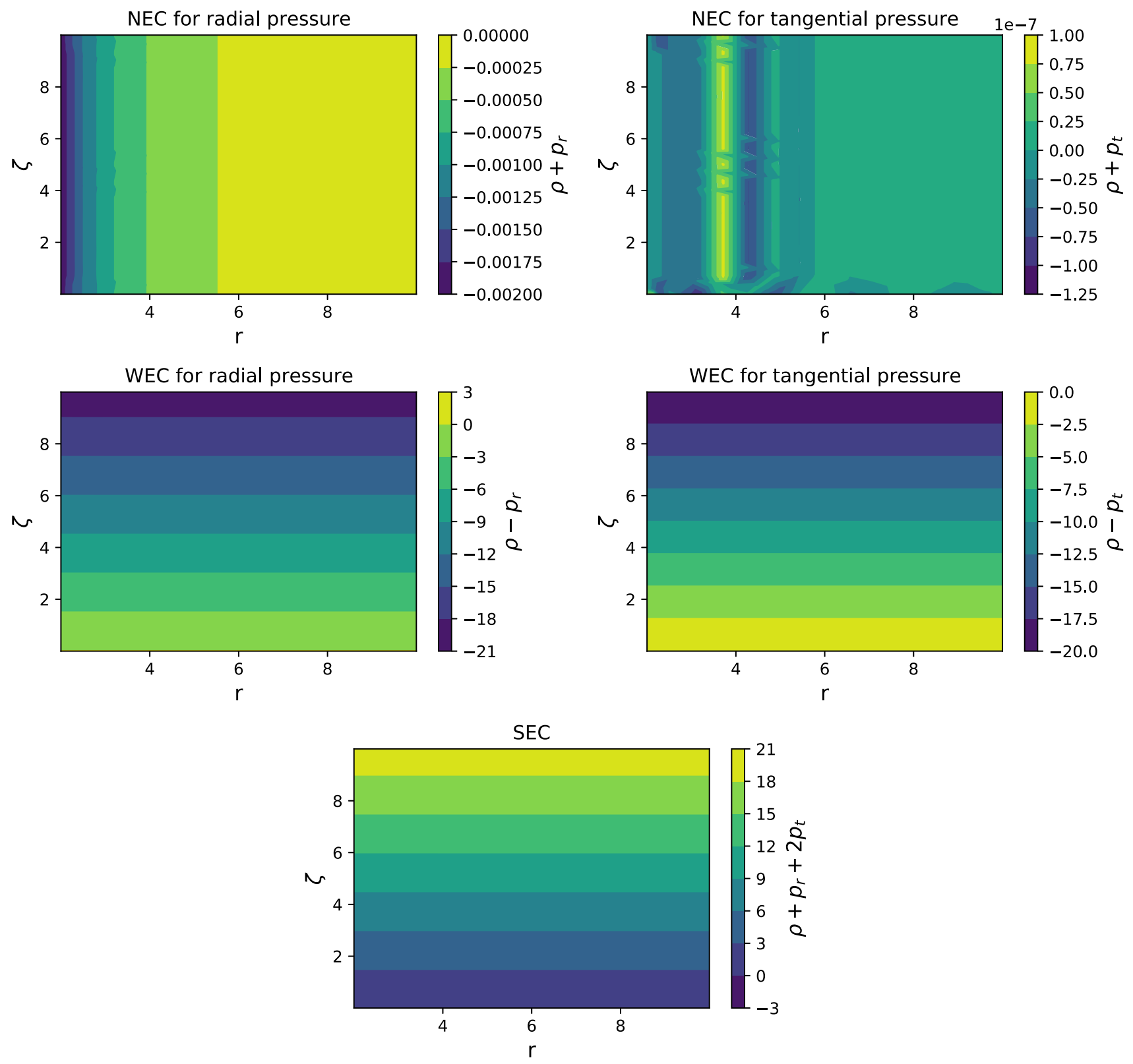

Fig. 9 NEC, WEC and SEC conditions for hybrid metric-Palatini gravity with $\omega=-1, \Phi=1$ and $k=\pi / 2$

\section{Quantization of exotic matter, that violate NEC condition}

Volume integral quantifier (further - just VIQ) could help us with the derivation of exact exotic matter volume. With VIQ we have the opportunity to understand with which values of the MOG parameters the volume of matter violating the null energy condition is the smallest in the case where exotic matter is present [32].

Volume integral quantifier is given by [42]:

$$
\begin{aligned}
\Phi= & \int_{r_{0}}^{\infty} \int_{0}^{\pi} \int_{0}^{2 \pi}\left[\rho+p_{r}\right] \sqrt{-g} d r d \theta d \phi \\
& \Downarrow \\
& \oint\left[\rho+p_{r}\right] d V=2 \int_{r_{0}}^{\infty}\left[\rho+p_{r}\right] 4 \pi r^{2} d r .
\end{aligned}
$$

From Fig. 10 obviously for any choice of MOG parameter if $r \rightarrow \infty$ then $\Phi \rightarrow 0$. Also, for any $\alpha>0 \wedge f_{0}>0 \wedge \zeta>0$ we have exotic matter at the throat, but we could minimize its amount in the first two MOG's of our consideration if we set $\alpha \rightarrow 0$ and $f_{0} \rightarrow 0$. For log-corrected gravity $\Phi$ is minimized at $\alpha=5$, for hybrid metric-Palatini gravity we 

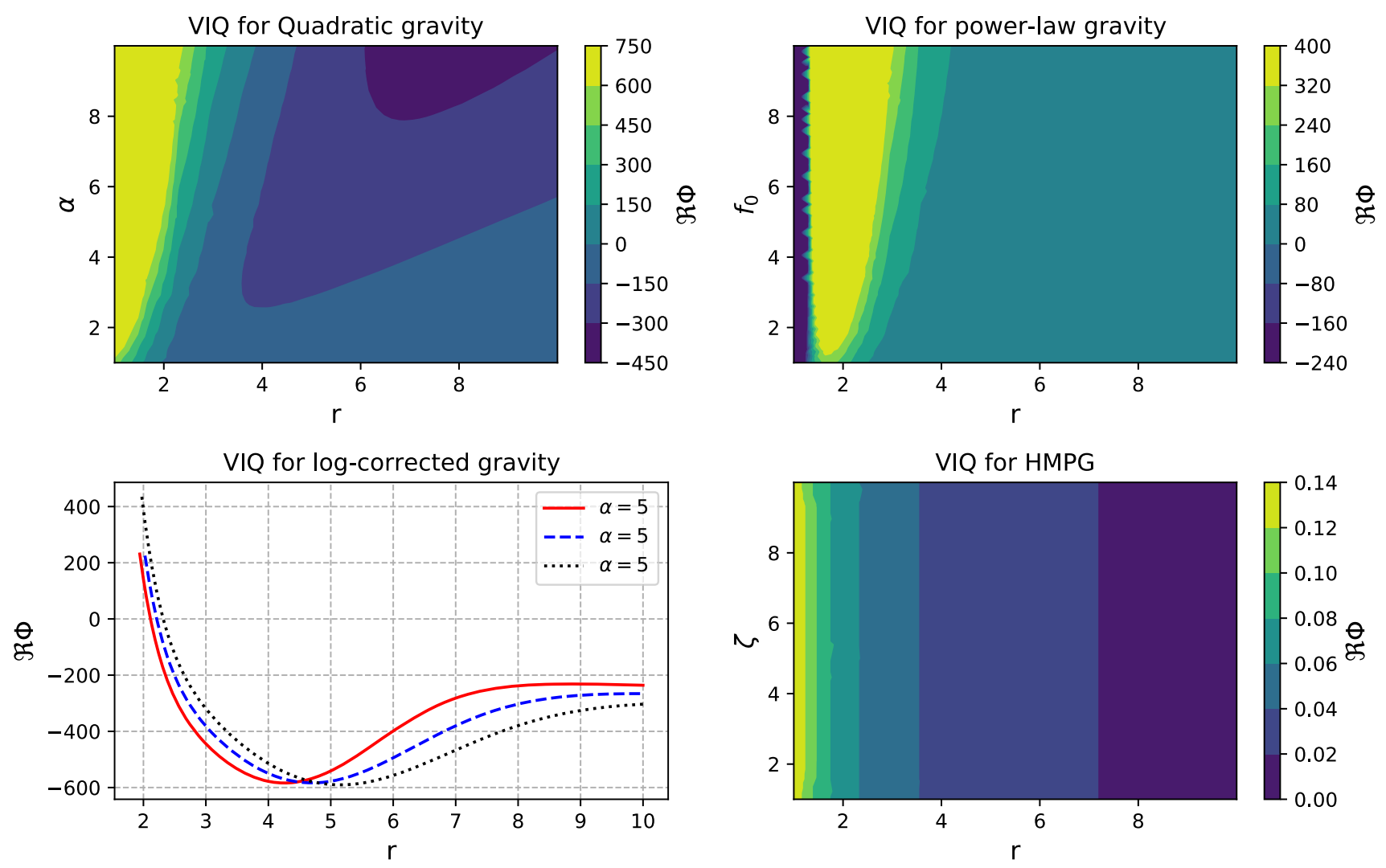

Fig. 10 Volume integral quantifier for quadratic, power-law, log-corrected and HMPG gravities. For the $f(\mathscr{R})$ MOG's we considered case with $\omega=1$ and for HMPG one with $\omega=-1$. Also, for both kinds of theories we assumed $r_{0}=1$

couldn't minimize exotic matter contribution because $\Phi$ is independent of $\zeta$ MOG parameter.

\section{Wormhole stability in modified theories of gravity}

Wormhole stability conditions can be examined by employing an equilibrium condition obtained from the TolmanOppenheimer-Volkov equation for non-tidal traversable wormhole: $[36,38,39,50]$ :

$$
\begin{array}{r}
\frac{d p_{r}}{d r}+\underbrace{\Omega(r)^{\prime}\left(\rho+p_{r}\right)}_{\text {if }} \Omega=0,0+\frac{2}{r}\left(p_{r}-p_{t}\right)+F_{\mathrm{ex}}=0 \\
\Downarrow \\
\frac{d p_{r}}{d r}+\frac{2}{r}\left(p_{r}-p_{t}\right)+F_{\mathrm{ex}}=0 .
\end{array}
$$

One may see that in our modified TOV (further - MTOV) present extra force $F_{\text {ex }}$, which exists to hold WH stable $[13,46]$, even if stress-energy tensor is not conserved (in considered theories of gravity generally $\left.\nabla^{\mu} T_{\mu \nu} \neq 0[29,30,43]\right)$.

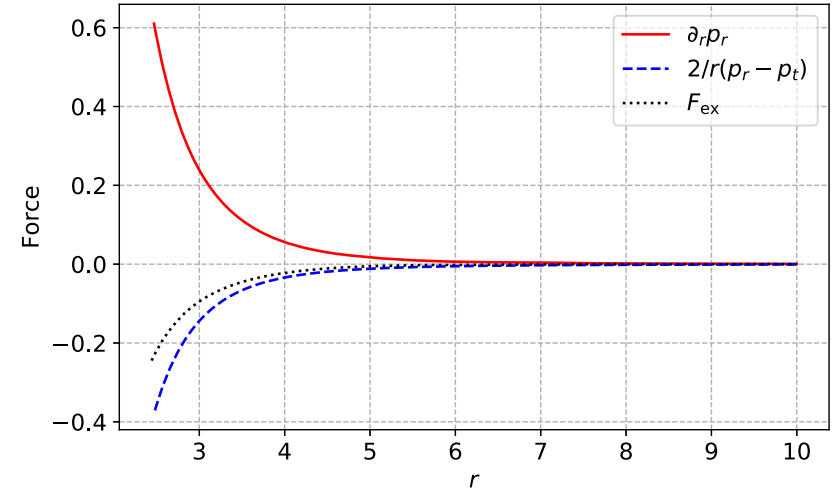

Fig. 11 MTOV forces example in quadratic gravity with $\alpha=\omega=1$

If the wormhole is stable, the MTOV conditions must be satisfied. Hence, for each model of our analysis it follows.

Routinely, on the Fig. 11 we have located the example of forces, that present in MTOV (45) for quadratic gravity case with $\alpha=\omega=1$.

$5.1 f(\mathscr{R})=\mathscr{R}+\alpha \mathscr{R}^{2}$ gravity

To satisfy MTOV, external force must look like: 
(1).pdf

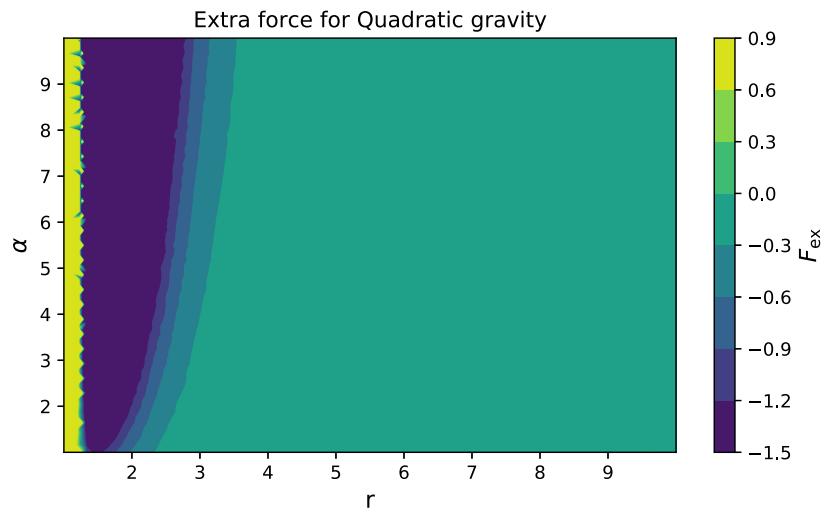

Fig. 12 MTOV extra force with varying $\alpha$ for $\omega=1$

$$
\begin{aligned}
F_{\mathrm{ex}}= & {\left[2 \alpha \left(2 r^{3}(r-b(r)) b^{(4)}(r)-18 r b^{\prime}(r)^{2}+r(r(11 b(r)\right.\right.} \\
& \left.-8 r) b^{(3)}(r)-b^{\prime \prime}(r)\left(r\left(r b^{\prime \prime}(r)-16\right)+26 b(r)\right)\right)+b^{\prime}(r) \\
& \left.\left.\times\left(r\left(-3 r^{2} b^{(3)}(r)+14 r b^{\prime \prime}(r)-16\right)+30 b(r)\right)\right)\right] /\left[r^{6}\right] .
\end{aligned}
$$

On the Fig. 12 we numerically solved Eq. (46). Judging by data from the figure above, we could say that as $\alpha \rightarrow 0 \Rightarrow$ $F_{\text {ex }} \rightarrow 0$ (GR restored). On other hand, for non-zero MOG parameter to keep wormhole stable there is always must be present extra force.

\section{$5.2 f(\mathscr{R})=f_{0} \mathscr{R}^{n}$ gravity}

MTOV extra force for power law gravity have following form:

$$
\begin{aligned}
& F_{\mathrm{ex}}=\left[f_{0} 2^{n-2}(n-1) n\left(\frac{b^{\prime}(r)}{r^{2}}\right)^{n+1}(2(n-3)(n-2)\right. \\
& \quad \times r^{3}(r-b(r)) b^{\prime \prime}(r)^{3}+6(1-2 n) r b^{\prime}(r)^{4} \\
& \quad-b^{\prime}(r)^{3}\left(r\left(r\left(3 r b^{(3)}(r)+2(5-6 n) b^{\prime \prime}(r)\right)+8 n(2 n-3)\right)\right. \\
& \left.\quad+2\left(-8 n^{2}+6 n+5\right) b(r)\right)+(n-2) r^{2} b^{\prime}(r) b^{\prime \prime}(r) \\
& \quad \times\left(6 r(r-b(r)) b^{(3)}(r)+((12 n-13) b(r)+4(4-3 n) r) b^{\prime \prime}(r)\right) \\
& +r b^{\prime}(r)^{2}\left((5-3 n) r^{2} b^{\prime \prime}(r)^{2}+2((4(5-3 n) n-5) b(r)\right. \\
& \quad+2(n(6 n-13)+6) r) b^{\prime \prime}(r)+r\left(2 r(r-b(r)) b^{(4)}(r)\right. \\
& \left.\left.\left.\left.+b^{(3)}(r)((12 n-13) b(r)+4(4-3 n) r)\right)\right)\right)\right] /\left[b^{\prime}(r)^{5}\right] .
\end{aligned}
$$
13.

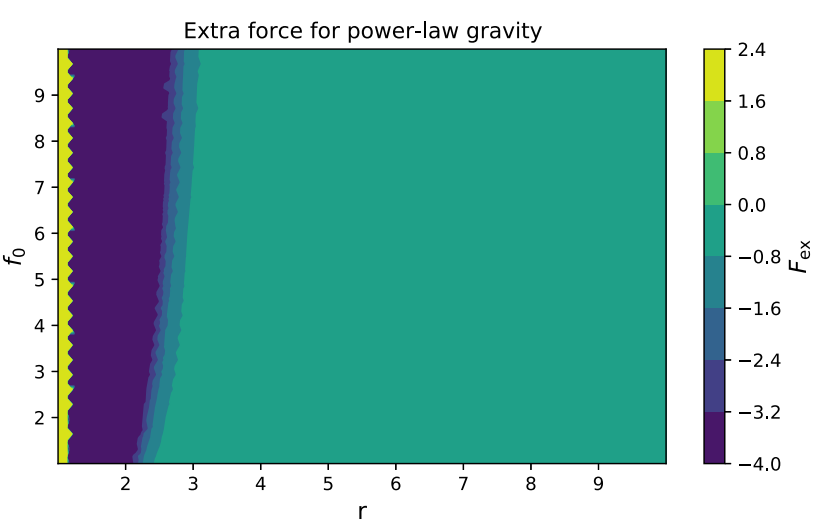

Fig. 13 MTOV extra force with varying $f_{0}$ for $\omega=1$

\section{$5.3 f(\mathscr{R})=\mathscr{R}+\alpha \mathscr{R}^{2}+\beta \mathscr{R}^{2} \ln \beta \mathscr{R}$ gravity}

Analytical solution for MTOV extra force in log-corrected gravity:

$$
\begin{aligned}
F_{\mathrm{ex}} & =\left[4 \beta r^{3}(b(r)-r) b^{\prime \prime}(r)^{3}-6 r b^{\prime}(r)^{4}\right. \\
& \times\left(6 \alpha+6 \beta \log \left(\frac{2 \beta b^{\prime}(r)}{r^{2}}\right)+13 \beta\right)+b^{\prime}(r)^{3}(2 b(r) \\
& \times\left(30 \alpha+30 \beta \log \left(\frac{2 \beta b^{\prime}(r)}{r^{2}}\right)+97 \beta\right) \\
& +r\left(r \left(b^{\prime \prime}(r)\left(28 \alpha+28 \beta \log \left(\frac{2 \beta b^{\prime}(r)}{r^{2}}\right)+66 \beta\right)\right.\right. \\
& \left.-3 r b^{(3)}(r)\left(2 \alpha+2 \beta \log \left(\frac{2 \beta b^{\prime}(r)}{r^{2}}\right)+3 \beta\right)\right) \\
& \left.\left.-32\left(\alpha+\beta \log \left(\frac{2 \beta b^{\prime}(r)}{r^{2}}\right)+4 \beta\right)\right)\right)+2 \beta r^{2} b^{\prime}(r) b^{\prime \prime}(r) \\
& \times\left(6 r(r-b(r)) b^{(3)}(r)+(11 b(r)-8 r) b^{\prime \prime}(r)\right)+r b^{\prime}(r)^{2} \\
& \times\left(-r^{2} b^{\prime \prime}(r)^{2}\left(2 \alpha+2 \beta \log \left(\frac{2 \beta b^{\prime}(r)}{r^{2}}\right)+9 \beta\right)+2 b^{\prime \prime}(r)\right. \\
& \times\left(2 \beta(8 r-13 b(r)) \log \left(\frac{2 \beta b^{\prime}(r)}{r^{2}}\right)-26 \alpha b(r)\right. \\
+ & (22 \alpha+57 \beta) b(r)-16 r(\alpha+3 \beta)))]\left[r^{6} b^{\prime}(r)^{2}\right] \\
& +95 \beta b(r)+16 \alpha r+68 \beta r)+r\left(2 r(r-b(r)) b^{(4)}(r)\right. \\
& \times\left(2 \alpha+2 \beta \log \left(\frac{2 \beta b^{\prime}(r)}{r^{2}}\right)+3 \beta\right) \\
& (3)(r)\left(2 \beta(11 b(r)-8 r) \log \left(\frac{2 \beta b^{\prime}(r)}{r^{2}}\right)\right.
\end{aligned}
$$

Therefore, as we did for other MOG's, we placed solution of Eq. (48) on the Fig. 14. Case with zero extra force (GR) could be obtained by setting $\alpha=\beta=0$. 


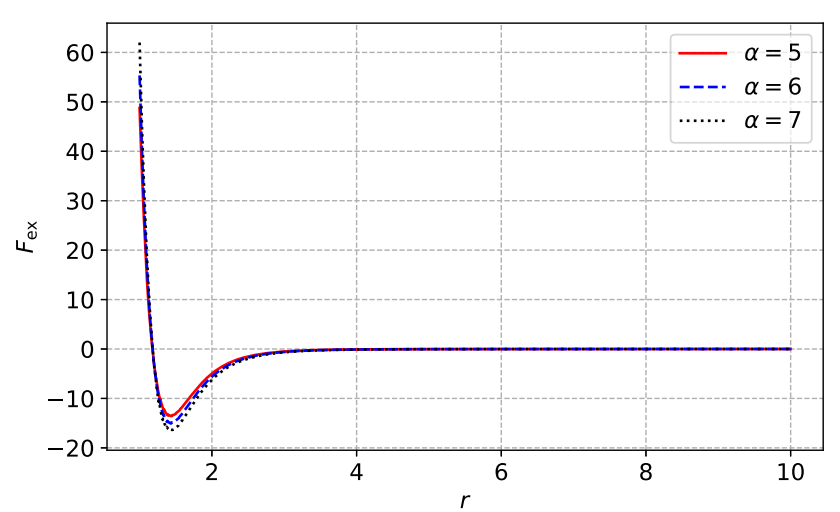

Fig. 14 MTOV extra force with varying $\alpha$ for $\beta=\omega=1$

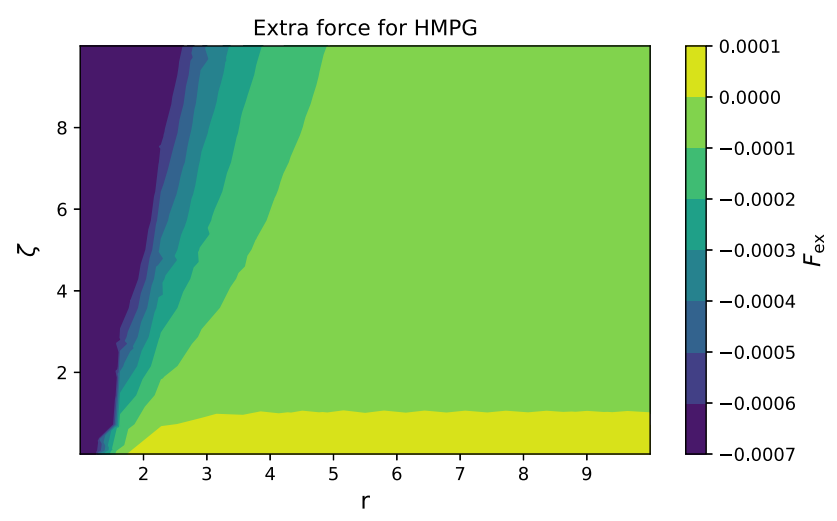

Fig. 15 MTOV extra force with varying $\zeta$ for $\Phi=1$ and $\omega=-1$

\subsection{Metric-Palatini exponential gravity}

For this MOG we have following $F_{\mathrm{ex}}$ :

$$
\begin{aligned}
& F_{\mathrm{ex}}=\left[\left[\operatorname { c s c } ^ { 2 } ( 2 \pi k ) \left(2 r b ( r ) \left(-2 r \cos ^{2}(2 \pi k) b^{\prime \prime}(r)+2 b^{\prime}(r)\right.\right.\right.\right. \\
& \left.\quad \times\left(\cos (4 \pi k)+r^{2}+1\right)+r^{2}-1\right)+b(r)^{2}\left(r \left((\cos (4 \pi k)+1) b^{\prime \prime}(r)\right.\right. \\
& \left.-4 r)-2 b^{\prime}(r)\left(\cos (4 \pi k)+r^{2}+1\right)\right)+r^{2}(r(\cos (4 \pi k)+1) \\
& \left.\left.\left.\times b^{\prime \prime}(r)-2 b^{\prime}(r)\left(\cos (4 \pi k)+r^{2}\right)\right)+2 r b(r)^{3}\right)\right] /\left[(r-b(r))^{2}\right] \\
& \left.+\frac{2 \zeta e^{-\frac{2 b^{\prime}(r)}{r^{2} \Phi}}\left(r b^{\prime \prime}(r)-2 b^{\prime}(r)\right)}{\Phi}\right] /\left[2 r^{3}\right] .
\end{aligned}
$$

Finally, we numerically solved Eq. (49) for last MOG of our consideration (HMPG) at the Fig. 15.

\section{Conclusions}

We presented Morris-Thorne traversable wormhole solutions for different modified gravity theories, such as: $f(\mathscr{R})=$ $\mathscr{R}+a \mathscr{R}^{2}, f(\mathscr{R})=f_{0} \mathscr{R}^{n}, f(\mathscr{R})=\mathscr{R}+\alpha \mathscr{R}^{2}+\beta \mathscr{R}^{2} \ln \beta \mathscr{R}$ and hybrid metric-Palatini gravity $f(\hat{\mathscr{R}})$. For each kind of modified gravity we derived suitable shape function that satisfies Einstein Field Equation by applying EoS $p_{t}=\omega \rho$. Numerical solutions for $b(r)$ and $b^{\prime}\left(r_{0}\right)$ are represented at Figs. 2, 4, 6 and 8.

We probed these models via numerical solutions of the null energy, weak energy and strong energy conditions, for $f(\mathscr{R})$ family of gravity theories results are presented at Figs. 3, 5 and 7, for metric-Palatini gravity at Fig. 9.

Moreover, we found a volume integral quantifier. The latter was used to construct plots with a volume of matter that violates NEC condition, i.e. exotic matter, and to obtain some parameter values and conditions, which could reduce the amount of exotic matter near the wormhole throat. The results of matter quantifying can be recognized in Fig. 10. As well, we probed the stability of the non-tidal wormholes in the modified gravities by the modified Tolman-OppenheimerVolkov equation (equilibrium). Furthermore, we found the contribution of the extra force that arises because of the noncontinuity of stress-energy tensor for each MOG w.r.t. free parameters. For the graphical representation of MTOV extra force solutions, see Figs. 12, 13, 14 and 15. More information about wormhole stability and suitable parameter values could be found in Sect. 5 .

This study contributes to the subject of the existence of wormhole solutions in modified theories of gravity. For the two fourth-order theories of our consideration, we found that the HMPG provides wormhole solutions with a fluid source with value for the EoS close to the cosmological constant. In a future study, we plan to investigate the relation of these solutions under the action of the conformal transformation which relates the two theories.

Data Availability Statement This manuscript has no associated data or the data will not be deposited. [Authors' comment: This work is fully theoretical, and thus no experimental data was used. The code could be provided on the request.]

Open Access This article is licensed under a Creative Commons Attribution 4.0 International License, which permits use, sharing, adaptation, distribution and reproduction in any medium or format, as long as you give appropriate credit to the original author(s) and the source, provide a link to the Creative Commons licence, and indicate if changes were made. The images or other third party material in this article are included in the article's Creative Commons licence, unless indicated otherwise in a credit line to the material. If material is not included in the article's Creative Commons licence and your intended use is not permitted by statutory regulation or exceeds the permitted use, you will need to obtain permission directly from the copyright holder. To view a copy of this licence, visit http://creativecomm ons.org/licenses/by/4.0/.

Funded by $\mathrm{SCOAP}^{3}$. 


\section{References}

1. K. Atazadeh, F. Darabi, Energy conditions in $\mathrm{f}(\mathrm{R} ; \mathrm{G})$ gravity. Gen. Relativ. Gravit. 46, 1664 (2014). https://doi.org/10.1007/ s10714-014-1664-8. arXiv:1302.0466 [gr-qc]

2. K.A. Bronnikov, Scalar-tensor theory and scalar charge. Acta Phys. Polon. B 4, 251-266 (1973)

3. K.A. Bronnikov, S.V. Bolokhov, M.V. Skvortsova, Hybrid metricPalatini gravity: black holes, wormholes, singularities, and instabilities. Gravit. Cosmol. 26(3), 212-227 (2020). https://doi.org/ 10.1134/S0202289320030044. ISSN:1995-0721

4. K.A. Bronnikov, M.V. Skvortsova, A.A. Starobinsky, Notes on wormhole existence in scalar-tensor and $\mathrm{F}(\mathrm{R})$ gravity. Gravit. Cosmol. 16(3), 216-222 (2010). https://doi.org/10.1134/ S0202289310030047. ISSN: 1995-0721

5. D.J. Brooker, S.D. Odintsov, R.P. Woodard, Precision predictions for the primordial power spectra from $f(R)$ models of inflation. Nucl. Phys. B 911, 318-337 (2016). https://doi.org/10.1016/ j.nuclphysb.2016.08.010. arXiv:1606.05879 [gr-qc]

6. H.A. Buchdahl, Non-linear Lagrangians and cosmological theory. Mon. Not. R. Astron. Soc. 150, 1 (1970)

7. S. Capozziello, M. De Laurentis, Extended theories of gravity. Phys. Rep. 509(4), 167-321 (2011). ISSN: 0370-1573. https://doi. org/10.1016/j.physrep.2011.09.003. https://www.sciencedirect. com/science/article/pii/S0370157311002432

8. S. Capozziello et al., Cosmology of hybrid metric-Palatini $\mathrm{f}(\mathrm{X})$ gravity. J. Cosmol. Astropart. Phys. 04, 011 (2012). https://doi. org/10.1088/1475-7516/2013/04/011

9. S. Capozziello et al., Hybrid modified gravity unifying local tests, galactic dynamics and latetime cosmic acceleration. Int. J. Mod. Phys. D 22, 1342006 (2013). https://doi.org/10.1142/ S0218271813420066

10. S. Carloni et al., Cosmological dynamics of Rn gravity. Class. Quantum Gravity 22, 4839-4868 (2005). https://doi.org/10.1088/ 0264-9381/22/22/011

11. N. Dadhich et al., $R=0$ spacetimes and selfdual Lorentzian wormholes. Phys. Rev. D 65, 064004 (2002). https://doi.org/10.1103/ PhysRevD.65.064004

12. B. Danila et al., Spherically symmetric static vacuum solutions in hybrid metric-Palatini gravity. Phys. Rev. D 99, 064028 (2019)

13. S. Dey, A. Chanda, B. Paul, Compact objects in $f(R, T)$ gravity with Finch-Skea geometry. Eur. Phys. J. Plus (2021). https://doi. org/10.1140/epjp/s13360-021-01173-w

14. E. Di Valentino et al., In the realm of the hubble tension-a review of solutions. Astrophysics. arXiv:2103.01183 [astro-ph.CO]

15. A. Einstein, N. Rosen, The particle problem in the general theory of relativity. Phys. Rev. 48, 73-77 (1935). https://doi.org/10.1103/ PhysRev.48.73

16. H. Ellis, Ether flow through a drainhole: A particle model in general relativity. J. Math. Phys. (1973). https://doi.org/10.1063/1. 1666161

17. T. Harko, F.S.N. Lobo, Beyond Einstein's general relativity: hybrid metric-Palatini gravity and curvature-matter couplings. Int. J. Mod. Phys. D 29(13), 2030008 (2020). https://doi.org/10.1142/ S0218271820300086

18. T. Harko et al., Metric-Palatini gravity unifying local constraints and late-time cosmic acceleration. Phys. Rev. D (2012). https://doi. org/10.1103/PhysRevD.85.084016

19. T. Harko et al., Metric-Palatini gravity unifying local constraints and late-time cosmic acceleration. Phys. Rev. D 85, 084016 (2012). https://doi.org/10.1103/PhysRevD.85.084016. arXiv: 1110.1049 [gr-qc]

20. D. Hochberg, T.W. Kephart, Wormhole cosmology and the horizon problem. Phys. Rev. Lett. 70, 2665-2668 (1993). https://doi.org/ 10.1103/PhysRevLett.70.2665
21. Q.-G. Huang, A polynomial $f(R)$ inflation model. JCAP 02, 035 (2014). https://doi.org/10.1088/1475-7516/2014/02/035. arXiv:1309.3514 [hep-th]

22. O. Ikram, G. Mustafa, Wormhole models and energy conditions in f gravity with the $\mathrm{Hu}-\mathrm{Sawicki}$ model. Adv. Astron. (2020). https:// doi.org/10.1155/2020/8813611

23. K. Jusufi, P. Channuie, M. Jamil, Traversable wormholes supported by GUP corrected Casimir energy. Eur. Phys. J. C (2020). https:// doi.org/10.1140/epjc/s10052-020-7690-7

24. S.-W. Kim, H. Lee, Exact solutions of a charged wormhole. Phys. Rev. D 63, 064014 (2001). https://doi.org/10.1103/PhysRevD.63. 064014

25. M.K. Zangeneh, F.S.N. Lobo, Dynamic wormhole geometries in hybrid metric-Palatini gravity. Eur. Phys. J. C 81(4), 285 (2021). https://doi.org/10.1140/epjc/s10052-021-09059-y. ISSN: $1434-6052$

26. P.K.F. Kuhfittig, Axially symmetric rotating traversable wormholes. Phys. Rev. D 67, 064015 (2003). https://doi.org/10.1103/ PhysRevD.67.064015. arXiv:grqc/0401028

27. F.S.N. Lobo, M.A. Oliveira, Wormhole geometries in $\mathrm{f}(\mathrm{R})$ modified theories of gravity. Phys. Rev. D 80, 104012 (2009). https://doi.org/ 10.1103/PhysRevD.80.104012. arXiv:0909.5539 [gr-qc]

28. C.F. Martins, P. Salucci, Analysis of rotation curves in the framework of Rn gravity. Mon. Not. R. Astron. Soc. 381, 1103-1108 (2007)

29. S.K. Maurya, F. Tello-Ortiz, Anisotropic fluid spheres in the framework of $f(R, T)$ gravity theory. Ann. Phys. 414, 168070 (2020). https://doi.org/10.1016/j.aop.2020.168070. https:// www.sciencedirect.com/science/article/pii/S0003491620300038. ISSN: 0003-4916

30. D. Momeni et al., Tolman-Oppenheimer-Volkoff equations in nonlocal f (R) gravity. Int. J. Mod. Phys. A 30(16), 1550093 (2015). https://doi.org/10.1142/S0217751X15500931. arXiv:1502.05000 [gr-qc]

31. M.S. Morris, K.S. Thorne, Wormholes in spacetime and their use for interstellar travel: a tool for teaching general relativity. Am. J. Phys. 56(5), 395-412 (1988). https://doi.org/10.1119/1.15620

32. K.K. Nandi, Y. Zhang, K.B. Vijaya Kumar, Volume integral theorem for exotic matter. Phys. Rev. D 70, 127503 (2004)

33. S. Nojiri, S.D. Odintsov, V.K. Oikonomou, Modified gravity theories on a nutshell: inflation, bounce and late-time evolution. Phys. Rep. 692, 1-104 (2017). https://doi.org/10.1016/j.physrep.2017. 06.001. arXiv:1705.11098 [gr-qc]

34. S. Nojiri, S. Odintsov, Introduction to modified gravity and gravitational alternative for dark energy. Int. J. Geom. Methods Mod. Phys. (2006). https://doi.org/10.1142/S0219887807001928

35. G.J. Olmo, Palatini approach to modified gravity: $f(R)$ theories and beyond. Int. J. Mod. Phys. D 20, 413-462 (2011). https://doi.org/ 10.1142/S0218271811018925. arXiv:1101.3864 [gr-qc]

36. J.R. Oppenheimer, G.M. Volkoff, On massive neutron cores. Phys. Rev. 55, 374-381 (1939). https://doi.org/10.1103/PhysRev.55.374

37. A. Paliathanasis, New cosmological solutions in hybrid metricPalatini gravity from dynamical symmetries (2020)

38. J. Ponce de Leon, Limiting configurations allowed by the energy conditions. Gen. Relativ. Gravit. 25(11), 1123-1137 (1993). https://doi.org/10.1007/BF00763756. ISSN: 1572-9532

39. F. Rahaman et al., Possible existence of wormholes in the galactic halo region. Eur. Phys. J. C 72(2), 2750 (2014). https://doi.org/10. 1140/epjc/s10052-014-2750-5. ISSN: 1434-6052

40. J. Sadeghi, H. Farahani, Logarithmic corrected F(R) gravity in the light of Planck 2015. Phys. Lett. B 751, 8995 (2015). ISSN: 0370-2693. https://doi.org/10.1016/j.physletb. 2015.10.020. https://www.sciencedirect.com/science/article/pii/ S0370269315007716 
41. P. Sahoo, A. Kirschner, P.K. Sahoo, Phantom fluid wormhole in $\mathrm{f}(\mathrm{R}$, T) gravity. Mod. Phys. Lett. A 37(37), 1950303 (2019). https://doi. org/10.1142/S0217732319503036

42. P. Sahoo, A. Kirschner, P.K. Sahoo, Phantom fluid wormhole in $\mathrm{f}(\mathrm{R}$, T) gravity. Mod. Phys. Lett. A 34(37), 1950303 (2019). https://doi. org/10.1142/S0217732319503036

43. I.G. Salako et al., Study on anisotropic strange stars in $\mathrm{f}(\mathrm{T}, \mathrm{T})$ gravity. Universe 6.10 (2020). ISSN: 2218-1997. https://doi.org/10. 3390/universe6100167. https://www.mdpi.com/2218-1997/6/10/ 167

44. J. Santos, Energy conditions in $\mathrm{f}(\mathrm{R})$-gravity. Phys. Rev. D 76, 083513 (2007). https://doi.org/10.1103/PhysRevD.76.083513. arXiv:0708.0411 [astro-ph]

45. M. Farasat Shamir, I. Fayyaz, Traversable wormhole solutions in f(R) gravity via Karmarkar condition. Eur. Phys. J. C 80(12), 1102 (2020). https://doi.org/10.1140/epjc/s10052-020-08689-y. ISSN: 1434-6052

46. Ksh Newton Singh et al., Exploring physical properties of compact stars in $\mathrm{f}(\mathrm{R} ; \mathrm{T})$ gravity: an embedding approach. Chin. Phys. C (2020). https://doi.org/10.1088/1674-1137/abab88

47. O. Sokoliuk, O. Baransky, Solving numerically EFE's in nonperturbed and linearly perturbed flat Kasner, Kasner-de Sitter spacetime with $R, f(R)$ and modified Gauss-Bonnet gravity and in asymptotically Kasner-like BKL singularity (2021). https://doi. org/10.13140/RG.2.2.16216.62723

48. T.P. Sotiriou, V. Faraoni, $\mathrm{f}(\mathrm{R})$ theories of gravity. Rev. Mod. Phys. 82, 451-497 (2010). https://doi.org/10.1103/RevModPhys. 82.451. arXiv:0805.1726 [gr-qc]
49. A.A. Starobinsky, A new type of isotropic cosmological models without singularity. Phys. Lett. B 91, 99-102 (1980). https://doi. org/10.1016/0370-2693(80)90670-X

50. R.C. Tolman, Static solutions of Einstein's field equations for spheres of fluid. Phys. Rev. 55, 364-373 (1939). https://doi.org/ 10.1103/PhysRev.55.364

51. M. Visser, Lorentzian wormholes: from Einstein to Hawking. ISBN: 978-1-56396-653-8 (1995)

52. M. Visser, Traversable wormholes: some simple examples. Phys. Rev. D 39, 3182-3184 (1989). https://doi.org/10.1103/PhysRevD. 39.3182

53. M. Visser, Traversable wormholes: the Roman ring. Phys. Rev. D 55, 5212-5214 (1997). https://doi.org/10.1103/PhysRevD.55. 5212

54. Y.B. Zeldovich, A hypothesis, unifying the structure and the entropy of the Universe. Mon. Not. R. Astron. Soc. 160, 1P (1972). https://doi.org/10.1093/mnras/160.1.1P 